\title{
On quadratic two-parameter families of spheres and their envelopes
}

\author{
Martin Peternell Boris Odehnal Maria Lucia Sampoli
}

December 7, 2007

\begin{abstract}
In the present paper we investigate rational two-parameter families of spheres and their envelope surfaces in Euclidean $\mathbb{R}^{3}$. The four dimensional cyclographic model of the set of spheres in $\mathbb{R}^{3}$ is an appropriate framework to show that a quadratic triangular Bézier patch in $\mathbb{R}^{4}$ corresponds to a two-parameter family of spheres with rational envelope surface. The construction shows also that the envelope has rational offsets. Further we outline how to generalize the construction to obtain a much larger class of surfaces with similar properties.
\end{abstract}

Keywords: Quadratic triangular Bézier surface; space of spheres; envelope of spheres, cyclographic image, linear congruence of lines, fibration; chordal variety.

\section{Introduction}

The rationality of envelopes of one- and two-parameter families of spheres has attracted the interest of several researchers, see [19, 20, 21, 22, 23, 24, 26, 33]. Unfortunately, the direct computation does in general not lead to rational parameterizations in case of rational envelopes and thus it is necessary to study these objects in more detail in order to gain insight to their geometric properties. The rationality of surfaces is determined by vanishing genus and second pluri-genus. Since the computation of these invariants is quite complex for envelopes given by parametric representations, the ascertainment of the rationality for the considered surfaces is difficult.

It has been proved in [26] that the non developable envelope of a rational one-parameter family of cones of revolution possesses rational parameterizations. Since any cone of revolution is the envelope of a one-parameter family of spheres as well as planes, the envelope 
is also generated by a two-parameter family of spheres. Using the affine space $\mathbb{R}^{4}$ as cyclographic model of the four-dimensional manifold of spheres in Euclidean $\mathbb{R}^{3}$, the mentioned result reads as: Any rational ruled surface in the model space $\mathbb{R}^{4}$ defines an envelope of a two-parameter system of spheres which possesses rational parameterizations and these parameterizations can be constructed explicitly. This result is a general statement about a class of surfaces in $\mathbb{R}^{4}$ and their corresponding envelopes in $\mathbb{R}^{3}$.

The question of rationality of envelopes is closely related to PH-curves and surface classes with similar properties. PH-curves have been introduced by Farouki, see the survey [10], and can be defined as planar polynomial curves possessing rational offset curves. The relation between offsets and envelopes is made obvious by the fact that the offset $C_{d}$ of a curve $C$ is the envelope of a one-parameter family of circles of constant radius $d$ centered at the curve $C$. Polynomial PH-curves possess rational arc length as an additional property. The rationality of the offset is equivalent to the existence of a rational unit normal vector field along the curve.

The large amount of contributions to PH-curves, see for instance $[5,6,7,8,9,10,11,12$, $16,17,25,29,30,37,38]$, which is being far from a complete list, already indicates that the curve case is quite well understood and applies even to practical design problems in NC-machining, see e.g. [40, 41]. The concept of PH-curves is also generalized to space curves, see e.g. [7, 10, 11, 38].

Rational surfaces with rational offsets are more involved, since the techniques for the curve case cannot be applied directly to surfaces. Although an explicit representation of all rational surfaces with rational offsets has been given already in [29], it is not obvious how to decide the rationality for particular surface classes. It has been proved that rational pipe surfaces [21], rational ruled surfaces [32] and all regular quadrics [24] possess rational offsets. These statements can also be found in [26] as specializations of a more general result concerning envelopes of one-parameter families of cones of revolution. Later it has been proved in $[14,15]$ that rational surfaces with linear normal vector fields, so called LN-surfaces, possess rational offset surfaces.

Using the cyclographic model $\mathbb{R}^{4}$ as framework, the main contribution of this article is to show that any quadratic triangular Bézier surface $\Phi$ in $\mathbb{R}^{4}$ corresponds to a two-parameter family of spheres with rational envelope. An immediate consequence is the rationality of the offset surfaces of the envelope. The proof is based on the study of the configuration of tangent planes and normal planes of surfaces $\Phi$. Quadratic triangular Bézier surfaces are thus examples for so called MOS-surfaces which have been introduced in [18]. The quadratic parameterization does not show this property immediately but it can be verified by applying the reparameterization proposed in Section 3. Further we outline a possible generalization to obtain a much larger family of surfaces in $\mathbb{R}^{4}$ which possesses similar properties concerning the rationality of the envelope of the corresponding family of spheres.

The paper is organized as follows: Section 2 explains some geometric properties of the space of spheres, in particular the cyclographic model and the computation of envelopes. 
Additionally we introduce to some concepts of line geometry which are used for the reparameterization technique. Further this section provides some basic properties of quadratic triangular Bézier surfaces in $\mathbb{R}^{4}$ and the structure of their tangent planes. Section 3 tells about the rational construction of the envelope surfaces of two-parameter families of spheres corresponding to quadratic triangular Bézier surfaces. Some examples illustrate the method. Further in Section 4 we report on a possible generalization of the obtained results concerning rationality of the envelope. Finally we conclude in Section 5 with some outlook to future research.

\section{Geometric background}

Points in $\mathbb{R}^{n}$ are represented by their coordinate vectors $\mathbf{x}=\left(x_{1}, \ldots, x_{n}\right)$. The projective closure of $\mathbb{R}^{n}$ is denoted by $\mathbb{P}^{n}$ and points $X$ in $\mathbb{P}^{n}$ are identified with their homogeneous coordinate vectors

$$
\mathbf{y} \mathbb{R}=\left(y_{0}, y_{1}, \ldots, y_{n}\right) \mathbb{R}=\left(y_{0}: y_{1}: \ldots: y_{n}\right), \text { with } \mathbf{y} \neq \mathbf{o .}
$$

Choosing the plane at infinity $\omega$ as $x_{0}=0$, the interchange between homogeneous and Cartesian coordinates for points in $\mathbb{R}^{n}$ is realized by

$$
x_{1}=\frac{y_{1}}{y_{0}}, x_{2}=\frac{y_{2}}{y_{0}}, \ldots, x_{n}=\frac{y_{n}}{y_{0}} .
$$

Moreover, we consider the dual projective space $\mathbb{P}^{n \star}$, whose points are identified with the hyperplanes in $\mathbb{P}^{n}$. Let $e_{0}+e_{1} x_{1}+\ldots+e_{n} x_{n}=0$ be the equation of a hyperplane $E \in \mathbb{R}^{n}$. The homogeneous coordinate vector $\mathbb{R} \mathbf{e}=\mathbb{R}\left(e_{0}, \ldots, e_{n}\right)$ is identified with $E$. Using homogeneous coordinates $y_{i}$, the equation of $E$ reads $e_{0} y_{0}+e_{1} y_{1}+\ldots+e_{n} y_{n}=0$.

Later on we use some facts on line geometry in $\mathbb{P}^{3}$. For that reason we recall the notation of Plücker coordinates and mention some facts about special two-parameter families of lines. For more details we refer to [34]. In order to introduce coordinates in the set $\mathcal{L}$ of lines in $\mathbb{P}^{3}$ we assume that a line $L$ is spanned by two different points $P=\mathbf{p} \mathbb{R}$ and $Q=\mathbf{q} \mathbb{R}$. The Plücker coordinates $\left(L_{1}, \ldots, L_{6}\right)$ of $L$ are defined by

$$
\left(p_{0} q_{1}-p_{1} q_{0}, p_{0} q_{2}-p_{2} q_{0}, p_{0} q_{3}-p_{3} q_{0}, p_{2} q_{3}-p_{3} q_{2}, p_{3} q_{1}-p_{1} q_{3}, p_{1} q_{2}-p_{2} q_{1}\right) .
$$

The coordinates $L_{i}$ of $L$ are homogeneous and independent on the choice of the points $X$ and $Y$ on $L$. Thus, they can be interpreted as the coordinates of points $\mathbf{L} \mathbb{R}=\left(L_{1}, \ldots, L_{6}\right) \mathbb{R}$ in a projective space $\mathbb{P}^{5}$. In addition the $L_{i}$ 's satisfy the Plücker relation

$$
L_{1} L_{4}+L_{2} L_{5}+L_{3} L_{6}=0 .
$$

The reparameterization technique in section 3 uses two-parameter families of lines $\mathcal{H}$ which send a unique line $H \in \mathcal{H}$ through any generic point $X \in \mathbb{P}^{3}$. Families of lines sharing this property are called a fibration of $\mathbb{P}^{3}$. Besides the star of lines the following fibrations of $\mathbb{P}^{3}$ are known. For illustrations we refer to Figures 4, 5 and 6. 
Hyperbolic linear line congruence: This family of lines in $\mathbb{P}^{3}$ consists of those lines intersecting two real skew lines $A$ and $B$ which are called the axes of the congruence.

Elliptic linear line congruence: This family of lines in $\mathbb{P}^{3}$ consists of those lines intersecting a pair of skew and conjugate complex lines $A$ and $\bar{A}$. Some authors use the notation spread for this family.

Parabolic linear line congruence: It consists of a one-parameter family of pencils of lines in planes through the axis $A$ and with vertices on $A$. The correspondence of the carrier planes of the pencils and the vertices is a projective mapping.

Chordal variety: It consists of the chords of a spatial cubic $C$ in $\mathbb{P}^{3}$. This family contains also tangent lines of $C$ and lines connecting two conjugate complex points of $C$.

Special congruence of lines of type $(1, \mathbf{n})$ : Consider an algebraic curve $C$ of degree $n$ and a line $L$ intersecting $C$ in $n-1$ points. The family of lines intersecting both $C$ and $L$ form a fibration of $\mathbb{P}^{3}$. For any generic point $X \notin C, L$ there exists a plane $X \vee L$ intersecting $C$ in a further point $Y$ and $X Y$ is the unique line of the family passing through $X$. The notation $(1, n)$ denotes the number of lines passing through a generic point and the number of lines lying in a generic plane, see e.g. [42].

A low degree example of the last family is given by the lines meeting a conic $C$ and a line $L$, where $L$ intersects $C$ at one point, see Fig. 5. A further example is obtained by all lines meeting a spatial cubic $C$ and a chord $L$ of $C$. Any fibration has an exceptional set, i.e. there exists at most a one-parameter family of points where the line is not unique.

\subsection{The cyclographic model of the space of spheres}

A sphere $S:(\mathbf{x}-\mathbf{m})^{2}=r^{2}$ in Euclidean 3 -space $\mathbb{R}^{3}$ is uniquely determined by its center $\mathbf{m}$ and its radius $r$. Spheres can be oriented by using signed radii. The family of oriented spheres in $\mathbb{R}^{3}$ will be denoted by $\mathcal{S}$.

The coordinate vector $\mathbf{m}$ of the center of a sphere $S$ together with the signed radius $r$ comprises the coordinates of a point $\mathbf{s}=(\mathbf{m}, r)$ in the affine space $\mathbb{R}^{4}$. On the other hand any point $\mathbf{s}=(\mathbf{m}, r) \in \mathbb{R}^{4}$ corresponds to a sphere in $\mathbb{R}^{3}$ with center $\left(m_{1}, m_{2}, m_{3}\right)$ and radius $r$. We call $\mathbb{R}^{4}$ the cyclographic model of the family $\mathcal{S}$ of spheres in $\mathbb{R}^{3}$. The cyclographic mapping $\gamma$ establishes the correspondence between points $\mathbf{s}$ in $\mathbb{R}^{4}$ and spheres $S$ in $\mathbb{R}^{3}$,

$$
\gamma: \mathbb{R}^{4} \rightarrow \mathcal{S}, \quad \mathbf{s}=(\mathbf{m}, r) \mapsto S:(\mathbf{x}-\mathbf{m})^{2}=r^{2} .
$$

Since the study of the mapping $\gamma$ gives a lot of insight to the construction of envelopes of spheres, we present some fundamental properties. 

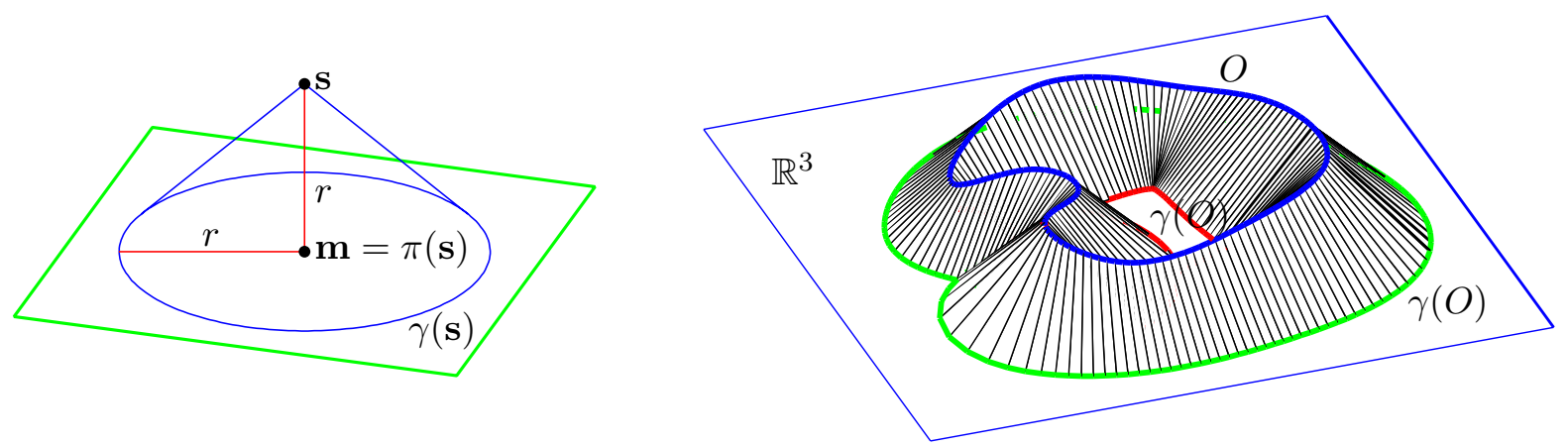

Figure 1: Left: Cyclographic mapping $\gamma: \mathbb{R}^{3} \rightarrow \mathbb{R}^{2}$. Right: Cyclographic image $\gamma(O) \in \mathbb{R}^{2}$ of curve $O \in \mathbb{R}^{3}$.

Let $\pi: \mathbb{R}^{4} \rightarrow \mathbb{R}^{3}$ be the orthogonal projection (top view) of points $\mathbf{y} \in \mathbb{R}^{4}$ to points $\pi(\mathbf{y})=\left(y_{1}, y_{2}, y_{3}\right)^{T}$ in $\mathbb{R}^{3}: x_{4}=0$. The sphere $\gamma(\mathbf{y})$ has center $\pi(\mathbf{y})$ and is given by the equation $\gamma(\mathbf{y}):(\mathbf{x}-\pi(\mathbf{y}))^{2}=y_{4}^{2}$.

We consider a geometric object $O$ in $\mathbb{R}^{4}$ and let $\gamma(O)$ be the corresponding family of spheres centered at the points of $\pi(O)$. If $\gamma(O)$ possesses a real envelope, we will use the notation $\gamma(O)$ as well for the envelope. The projection $\pi(O)$ is also called medial axis of the envelope $\gamma(O)$. Given $\pi(O)$ and the radius function of points $\in \pi(O)$, the envelope $\gamma(O)$ can be reconstructed from this information. The geometric object $O$ itself is also called medial axis transform.

\subsubsection{The cyclographic image of a line}

Considering a straight line $G: \mathbf{y}(t)=\mathbf{a}+t \mathbf{g}$ in $\mathbb{R}^{4}$, its points correspond to spheres whose centers $\mathbf{m}(t)$ trace the line $\pi(G)$. In general, the envelope is a cone of revolution with axis $\pi(G)$, see Fig 2. Considering a line $G$ with horizontal direction vector $\mathbf{g}=\left(g_{1}, g_{2}, g_{3}, 0\right)^{T}$, the envelope $\gamma(G)$ is a cylinder of revolution, with axis $\pi(G)$ and radius $a_{4}$. If we choose $G: \mathbf{y}(t)=\mathbf{a}+t(0,0,0,1)^{T}$, the image $\gamma(\mathbf{y}(t))$ is the family of concentric spheres centered at $\mathbf{m}=\left(a_{1}, a_{2}, a_{3}\right)^{T}$, which does not possess a real envelope. The lines $G$ with direction vector $\mathbf{g}=\left(g_{1}, g_{2}, g_{3}, g_{4}\right)$ satisfying $\langle\mathbf{g}, \mathbf{g}\rangle \geq 0$ are related to real envelopes $\gamma(G)$, where the scalar product $\langle$,$\rangle is defined by$

$$
\langle\mathbf{x}, \mathbf{x}\rangle:=\mathbf{x}^{T} D \mathbf{x}, \text { with } D=\operatorname{diag}(1,1,1,-1) .
$$

Equipping $\mathbb{R}^{4}$ with the scalar product $\langle$,$\rangle yields a pseudo-Euclidean or Minkowski space.$ Some authors use the notion $\mathbb{R}^{3,1}$ to emphasize the signature of $D$. Lines $G$ are called Euclidean, isotropic or pseudo-Euclidean depending on whether the direction vector $\mathbf{g}$ satisfies $\langle\mathbf{g}, \mathbf{g}\rangle>0,\langle\mathbf{g}, \mathbf{g}\rangle=0$, or $\langle\mathbf{g}, \mathbf{g}\rangle<0$. The respective notations space-like, light-like or time-like are also commonly used. 

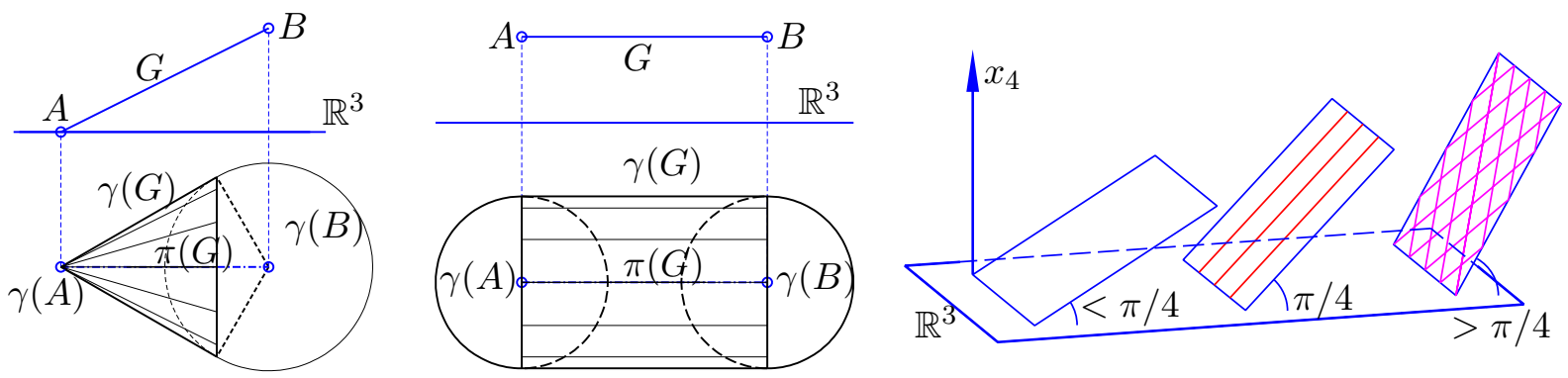

Figure 2: Left and middle: Cone and cylinder of revolution as cyclographic image of a line $\in \mathbb{R}^{4}$. Right: Three types of planes and their isotropic directions.

\subsubsection{The cyclographic image of a surface}

We briefly discuss the cyclographic image $\gamma(\Phi)$ of a plane $\Phi \in \mathbb{R}^{4}$ first, since it follows similar rules as the cyclographic image of a line. Let $\mathbf{f}(u, v)=\mathbf{a}+u \mathbf{g}+v \mathbf{h}$ be a linear parameterization of $\Phi$. If $\Phi$ is parallel to $\mathbb{R}^{3}: x_{4}=0$, the direction vectors $\mathbf{g}=\left(g_{1}, g_{2}, g_{3}, 0\right)$ and $\mathbf{h}=\left(h_{1}, h_{2}, h_{3}, 0\right)$ are horizontal. The envelope $\gamma(\Phi)$ consists of two parallel oriented planes having distance $\pm a_{4}$ from $\pi(\Phi)$. For planes $\Phi$ not parallel to $\mathbb{R}^{3}$ we have to distinguish the following cases, see Fig. 2.

A plane $\Phi \in \mathbb{R}^{4}$ is called Euclidean if all direction vectors $\mathbf{r}(u, v)=u \mathbf{g}+v \mathbf{h}$ are space-like. This means that $\langle\mathbf{r}, \mathbf{r}\rangle=0$ has no real zeros and $\gamma(\Phi)$ consists of two real planes $\Phi_{1}, \Phi_{2}$. A plane $\Phi$ is called isotropic if $\langle\mathbf{r}, \mathbf{r}\rangle=0$ has a double zero. The corresponding direction is the only isotropic direction in $\Phi$. A plane $\Phi$ is called pseudo-Euclidean if $\langle\mathbf{r}, \mathbf{r}\rangle=0$ has a two real zeros corresponding to two isotropic directions in $\Phi$. There is no real envelope but $\gamma(\Phi)$ consists of a pair of conjugate complex planes.

The cyclographic image $\gamma(\Phi)$ of a two-dimensional surface $\Phi$ with parameterization $\mathbf{f}: U \subset$ $\mathbb{R}^{2} \rightarrow \mathbb{R}^{4}$ is the envelope of the two-parameter family of spheres $S$ centered at the projection $\pi(\Phi)=\pi(\mathbf{f}(U))$ with radius function $r(u, v)=f_{4}(u, v)$. A Euclidean tangent plane $T$ at $F$ of $\Phi$ is mapped to a pair of planes touching the envelope $\gamma(\Phi)$ (and also the sphere $\gamma(\mathbf{f})$ ) at two points $F_{1}, F_{2}$.

As an important example we note that the cyclographic image $\gamma(\Phi)$ of a ruled surface $\Phi \in \mathbb{R}^{4}$ is the envelope of a one-parameter family of cones of revolution $\gamma(G)$ corresponding to the generating lines $G \in \Phi$.

We continue the discussion of the cyclographic image $\gamma(\Phi)$ of a surface $\Phi$ later in Section 2.1.4 where we take a projective viewpoint. Now we briefly study the envelope construction of a two parameter family of spheres in $\mathbb{R}^{3}$. 


\subsubsection{Envelope construction in the surface case}

Let us consider a surface $\Phi \in \mathbb{R}^{4}$, parameterized by $\mathbf{f}(u, v)$ and let us denote the top view $\pi(\mathbf{f})$ by $\mathbf{m}(u, v)$ and $r(u, v)=f_{4}(u, v)$. The envelope of the two-parameter family of spheres $S(u, v):(\mathbf{x}-\mathbf{m})^{2}=r^{2}$ can be obtained as solution of

$$
\begin{array}{lll}
S & :(\mathbf{x}-\mathbf{m})^{2}-r^{2} & =0 \\
S_{u}:(\mathbf{x}-\mathbf{m})^{T} \cdot \mathbf{m}_{u}+r r_{u} & =0 \\
S_{v} & :(\mathbf{x}-\mathbf{m})^{T} \cdot \mathbf{m}_{v}+r r_{v} & =0 .
\end{array}
$$

Since the partial derivatives $S_{u}:=\partial S / \partial u, S_{v}:=\partial S / \partial v$ are planes, the envelope contains the intersection points of the lines $g(u, v)=S_{u} \cap S_{v}$ and the spheres $S(u, v)$. In general $g \cap S$ consists of two points and thus the parameterization of the envelope is obtained by solving a quadratic equation. This direct approach leads to parameterizations containing square roots even if the cyclographic image admits rational parameterizations.

\subsubsection{Envelope construction using the projective extension in $\mathbb{R}^{4}$}

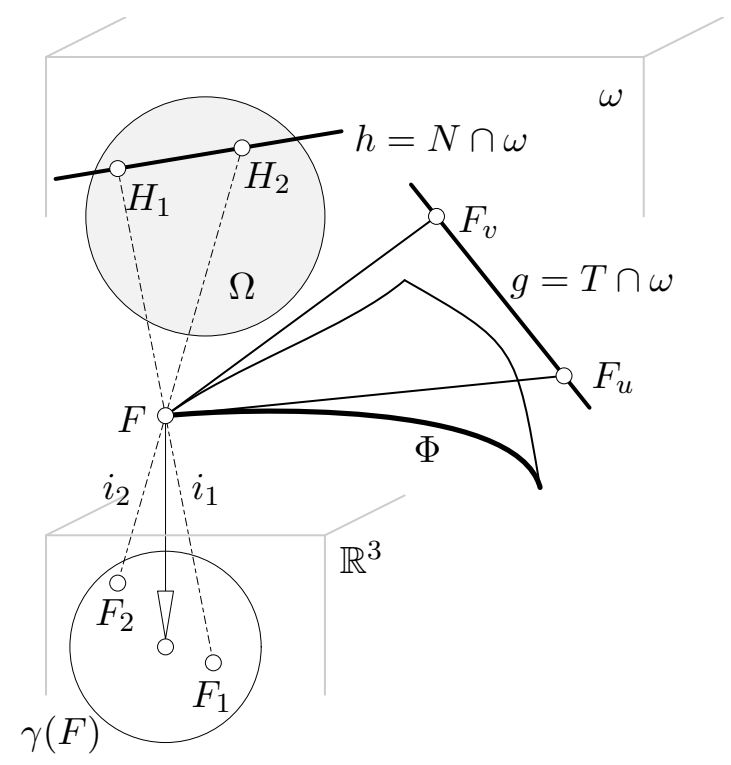

Figure 3: Construction of the cyclographic image from the projective point of view.

Embedding the Minkowski space $\mathbb{R}^{4}$ into projective 4-space $\mathbb{P}^{4}$, we use homogeneous coordinates $\left(y_{0}, y_{1}, \ldots, y_{4}\right) \mathbb{R}=\mathbf{y} \mathbb{R}$ for points $Y \in \mathbb{P}^{4}$. The projective 3 -space at infinity is given by $\omega: y_{0}=0$. The polar system of the quadric

$$
\Omega: y_{1}^{2}+y_{2}^{2}+y_{3}^{2}-y_{4}^{2}=0, y_{0}=0
$$


in $\omega$ defines the scalar product (5) whose coefficient matrix $D$ equals the coefficient matrix of the quadric $\Omega$. Orthogonality in $\mathbb{R}^{4}$ can also be defined with help of the polar system of $\Omega$ : Consider two lines $g$ and $h$ in $\mathbb{R}^{4}$. Their ideal points $G=g \cap \omega$ and $H=h \cap \omega$ are represented by $G=(0, \mathbf{g}) \mathbb{R}$ and $H=(0, \mathbf{h}) \mathbb{R}$ where $\mathbf{g}=\left(g_{1}, \ldots, g_{4}\right)$ and $\mathbf{h}=\left(h_{1}, \ldots, h_{4}\right)$ are some direction vectors of $g$ and $h$. If $\langle\mathbf{g}, \mathbf{h}\rangle=0$ holds, the lines $g$ and $h$ are orthogonal with respect to (5). This is equivalent to the polar position of $G$ and $H$ with respect to $\Omega$. Depending on whether the ideal point of a line is outside, or inside or contained in $\Omega$, the line is called space-like, or time-like or light-like.

The light-like lines $i$ through a point $P \in \mathbb{R}^{4}$ form the light-cone $\Gamma_{P}$ with vertex $P$,

$$
\Gamma_{P}:\langle\mathbf{x}-\mathbf{p}, \mathbf{x}-\mathbf{p}\rangle=0,
$$

where $\mathbf{x}=\left(x_{1}, \ldots, x_{4}\right)$ and $\mathbf{p}=\left(p_{1}, \ldots, p_{4}\right)$ are affine coordinates in $\mathbb{R}^{4}$. The intersection of the light-cone $\Gamma_{P}$ with $\mathbb{R}^{3}: y_{4}=0$ is exactly the non-oriented sphere $S_{p}:(\mathbf{x}-\pi(\mathbf{p}))^{2}-p_{4}^{2}=$ 0 . Thus the cyclographic mapping can be defined also via

$$
\gamma: \mathbb{R}^{4} \rightarrow \mathcal{S}, \quad P \mapsto S_{p}=\Gamma_{P} \cap \mathbb{R}^{3} .
$$

This definition of $\gamma$ is not fully equivalent to (4) from section 2.1 since $S_{p}=\Gamma_{P} \cap \mathbb{R}^{3}$ are not oriented spheres. When considering envelopes $\gamma(O)$ of families of spheres as oriented surfaces in $\mathbb{R}^{3}$, the orientation of the involved spheres can be added.

Let a surface $\Phi: \mathbf{f}(u, v)$ in $\mathbb{R}^{4}$ be given. The construction of the cyclographic image $\gamma(\Phi)$ is illustrated in Fig. 3 and can be performed as follows.

- The tangent planes $T(u, v)$ of points $\mathbf{f}(u, v)=F \in \Phi$ are intersected with $\omega: y_{0}=0$ and yield the two-parameter family of ideal lines $g(u, v)=T(u, v) \cap \omega$.

- The polarity $\lambda: \mathbb{P}^{3} \rightarrow \mathbb{P}^{3 \star}$ with respect to the quadric $\Omega$ maps $g(u, v)$ to lines $h(u, v)=\lambda(g(u, v)) \in \omega$. Since $\Omega$ induces the orthogonality in $\mathbb{R}^{4}$, the lines $h(u, v)$ are the ideal lines of the normal planes $N$ of $\mathbf{f}(u, v)=F \in \Phi$. Generically, any line $h(u, v)$ intersects $\Omega$ in two points $H_{1}(u, v)$ and $H_{2}(u, v)$.

- By joining $H_{1}$ and $H_{2}$ with the corresponding point $F \in \Phi$ one obtains the isotropic projection lines $i_{1}=H_{1} F$ and $i_{2}=H_{2} F$. The intersection points $F_{1}=i_{1} \cap \mathbb{R}^{3}$ and $F_{2}=i_{2} \cap \mathbb{R}^{3}$ of these projections lines $i_{1}$ and $i_{2}$ with $\mathbb{R}^{3}: y_{4}=0$ lead to a parameterization of the envelope $\gamma(\Phi)$.

The isotropic projection lines $i_{1}$ and $i_{2}$ are the intersections of the light-cone $\Gamma_{F}$ with the normal plane $N$ of $\Phi$ passing through $F$. These two-parameter family of lines $i_{1}, i_{2}$ form the isotropic hyper-surface $\Gamma(\Phi)$ passing through $\Phi$. This method of computing the cyclographic image has already been proposed in [20]. 
Analytically the isotropic hyper-surface $\Gamma(\Phi)$ is determined by the equations

$$
\begin{aligned}
\Gamma_{f}:\langle\mathbf{x}-\mathbf{f}, \mathbf{x}-\mathbf{f}\rangle & =0 \\
\frac{\partial \Gamma_{f}}{\partial u}:\left\langle\mathbf{x}-\mathbf{f}, \mathbf{f}_{u}\right\rangle & =0 \\
\frac{\partial \Gamma_{f}}{\partial v}:\left\langle\mathbf{x}-\mathbf{f}, \mathbf{f}_{v}\right\rangle & =0
\end{aligned}
$$

where $\mathbf{f}_{u}$ and $\mathbf{f}_{v}$ denote the partial derivatives of $\mathbf{f}(u, v)$. The first equation describes the light-cone through $\mathbf{f}$, the second and third equation describes the normal plane $N$. This system expresses also the envelope condition for the two-parameter family of light-cones $\Gamma_{F}(u, v)$. Comparing the system (10) with (6) we recognize that the envelope of the twoparameter system of spheres $S_{f}:(\mathbf{x}-\pi(\mathbf{f}))^{2}-f_{4}^{2}=0$ is the intersection $\Gamma \cap \mathbb{R}^{3}: x_{4}=0$ of the isotropic hyper-surface $\Gamma(\Phi)$ with $x_{4}=0$. The offset surface of $\gamma(\Phi)$ at distance $d$ is obtained by intersecting $\Gamma(\Phi)$ with $x_{4}=d$.

\subsection{Quadratic triangular Bézier surfaces}

Quadratic triangular Bézier surfaces in $\mathbb{R}^{4}$ can be represented with respect to barycentric coordinates $u, v, w=1-u-v$ by the parameterization

$$
\mathbf{s}(u, v, w)=\sum_{i+j+k=2} B_{i j k}^{2}(u, v, w) \mathbf{b}_{i j k}
$$

with basis functions $B_{i j k}^{2}=\frac{2 !}{i ! j ! k !} u^{i} v^{j} w^{k}$ and control points $\mathbf{b}_{i j k}$, see [13]. For our purposes it is more convenient to use a parameterization in monomial form with affine parameters $u, v$,

$$
\mathbf{s}(u, v)=\frac{1}{2} \mathbf{a}_{1} u^{2}+\mathbf{a}_{2} u v+\frac{1}{2} \mathbf{a}_{3} v^{2}+\mathbf{a}_{4} u+\mathbf{a}_{5} v+\mathbf{a}_{6}, \text { with } \mathbf{a}_{i} \in \mathbb{R}^{4} .
$$

Quadratic triangular Bézier surfaces $\Phi$ have attracted much interest in the past [39] and more recently $[1,2,3,4,28,36]$. From the parameterization (11) it follows that $S$ is obtained as projection of the Veronese surface $V_{2}^{2} \in \mathbb{R}^{5}$ with affine parameterization $\mathbf{v}(u, v)=\left(u^{2}, u v, v^{2}, u, v\right)$.

It will turn out that the structure of the tangent planes $T$ of a quadratic triangular Bézier surface $\Phi$ and the structure of their ideal lines $g=T \cap \omega$ is responsible for the rationality of the isotropic hyper-surface $\Gamma(\Phi)$ and therefore for the rationality of the cyclographic image $\gamma(\Phi)$. This compares to a similar result in $\mathbb{R}^{3}$ where the structure of the set of tangent planes of quadratic triangular Bézier surfaces is responsible for their LN-property, see [27]. 


\subsection{The tangent planes and normal planes of quadratic triangu- lar Bézier surfaces in $\mathbb{R}^{4}$}

We embed the quadratic triangular Bézier surface $\Phi \subset \mathbb{R}^{4}$ from (11) into $\mathbb{P}^{4}$. This is done by representing $\Phi$ by the homogeneous coordinates $\mathbf{f}(u, v) \mathbb{R}=(1, \mathbf{s}(u, v)) \mathbb{R}$ corresponding to (11). The tangent planes $T$ of the points $F=\mathbf{f} \mathbb{R}$ of $\Phi$ are spanned by $F$ and the derivative points

$$
\begin{aligned}
& F_{u}=\mathbf{f}_{u} \mathbb{R}=\left(0, \mathbf{s}_{u}\right) \mathbb{R}=\left(0, \mathbf{a}_{1} u+\mathbf{a}_{2} v+\mathbf{a}_{4}\right) \mathbb{R} \\
& F_{v}=\mathbf{f}_{v} \mathbb{R}=\left(0, \mathbf{s}_{v}\right) \mathbb{R}=\left(0, \mathbf{a}_{2} u+\mathbf{a}_{3} v+\mathbf{a}_{5}\right) \mathbb{R} .
\end{aligned}
$$

The ideal points $F_{u}=\mathbf{f}_{u} \mathbb{R}$ as well as $F_{v}=\mathbf{f}_{v} \mathbb{R}$ represent linear parameterizations of linear subspaces $E_{u}, E_{v} \subset \omega$. In case rk $\left(\mathbf{a}_{1}, \mathbf{a}_{2}, \mathbf{a}_{4}\right)=$ rk $\left(M_{u}\right)=3$ and rk $\left(\mathbf{a}_{2}, \mathbf{a}_{3}, \mathbf{a}_{5}\right)=$ rk $\left(M_{v}\right)=$ 3 both $E_{u}$ and $E_{v}$ are planes and the linear parameterizations of $F_{u}$ and $F_{v}$ induce a projective mapping $\alpha: F_{u} \in E_{u} \mapsto F_{v} \in E_{v}$ between $E_{u}$ and $E_{v}$.

Assuming that $\Phi$ spans $\mathbb{P}^{4}$ it follows that the family of lines $\mathcal{G}$ spans $\omega$. This implies that we can exclude cases where $\mathcal{G}$ is a ruled plane. Thus rk $\left(M_{u}\right) \geq 2$ and rk $M_{v} \geq 2$ holds. The case where $\Phi$ is contained in a 3 -space $\subset \mathbb{P}^{4}$ does not cause any problems and will be discussed at the end of this section.

According to the classification in [28] there exist five relevant cases of quadratic triangular Bézier surfaces in affine space $\mathbb{R}^{4} \subset \mathbb{P}^{4}$. Each class of surfaces is represented by a surface $\Phi$ parameterized in an affine normal form. The respective families $\mathcal{G}$ and $\mathcal{H}$ of ideal lines of tangent planes and normal planes are illustrated in figures 4, 5 and 6 . Additionally we give the algebraic equations of the families of lines $\mathcal{G}$ in terms of their Plücker coordinates $\mathbf{G}=\left(G_{1}, \ldots, G_{6}\right)$. The ideal points $\left(0, \mathbf{a}_{i}\right) \mathbb{R}$ corresponding to the vectors $\mathbf{a}_{i}$ in (12) are denoted by $A_{i}$.

Case 1: The parameterization of $\Phi$ is assumed to be $\mathbf{f}(u, v)=\left(\frac{1}{2} u^{2}, \frac{1}{2} v^{2}, u, v\right)$. Since $\mathbf{a}_{2}=(0,0,0,0)$ one obtains rk $\left(\mathbf{a}_{1}, \mathbf{a}_{2}, \mathbf{a}_{4}\right)=2$ and $\mathbf{r k}\left(\mathbf{a}_{2}, \mathbf{a}_{3}, \mathbf{a}_{5}\right)=2$. Thus $E_{u}$ and $E_{v}$ degenerate to a pair of skew lines. It follows that the family of ideal lines $\mathcal{G}$ forms a hyperbolic linear line congruence, see Fig. 4 , joining the points $F_{u}=\left(u \mathbf{a}_{1}+\mathbf{a}_{4}\right)$ and $F_{v}=\left(v \mathbf{a}_{3}+\mathbf{a}_{5}\right)$. The Plücker coordinates of lines $g$ are $\mathbf{G}=(u v, 0, u, 1,0,-v) \mathbb{R}$ and satisfy the two linear relations $G_{2}=0, G_{5}=0$.

The family $\mathcal{H}=\lambda(\mathcal{G})$ of polar lines $h(u, v)$ is a hyperbolic linear line congruence, too. This follows from the fact that the polar images $\lambda\left(E_{u}\right)$ and $\lambda\left(E_{v}\right)$ are two skew lines, not considered as range of points but as pencils of planes. The lines $h \in \mathcal{H}$ are obtained by intersecting two arbitrary planes of these pencils.

Case 2: The parameterization of $\Phi$ is assumed to be $\mathbf{f}(u, v)=\left(\frac{1}{2} u^{2}, u v, u, v\right)$. Therefore rk $\left(\mathbf{a}_{1}, \mathbf{a}_{2}, \mathbf{a}_{4}\right)=3$ and rk $\left(\mathbf{a}_{2}, \mathbf{a}_{3}, \mathbf{a}_{5}\right)=2$ which says that $E_{u}$ is a linearly parameterized plane and $E_{v}$ degenerates to a line. The family $\mathcal{G}$ consists of lines joining points 
$F_{v}(u)=\left(0, u \mathbf{a}_{2}+\mathbf{a}_{5}\right) \mathbb{R}$ and $F_{u}=\left(0, u \mathbf{a}_{1}+v \mathbf{a}_{2}+\mathbf{a}_{4}\right) \mathbb{R}$. These lines form a parabolic linear line congruence with axis joining $A_{2}=\left(0, \mathbf{a}_{2}\right) \mathbb{R}$ and $A_{5}=\left(0, \mathbf{a}_{5}\right) \mathbb{R}$. It decomposes into pencils of lines, contained in planes $\varepsilon(u)=F_{v} \vee A_{2} \vee\left(u \mathbf{a}_{1}+\mathbf{a}_{4}\right) \mathbb{R}$ with vertices $F_{v}$. The Plücker coordinates of the lines $g$ of the linear line congruence are $\mathbf{G}=\left(u^{2}, 0, u, 1,-v,-u\right) \mathbb{R}$ and satisfy the two linear relations $G_{2}=0, G_{3}+G_{6}=0$. The family $\mathcal{H}=\lambda(\mathcal{G})$ of polar lines is again a parabolic linear line congruence.
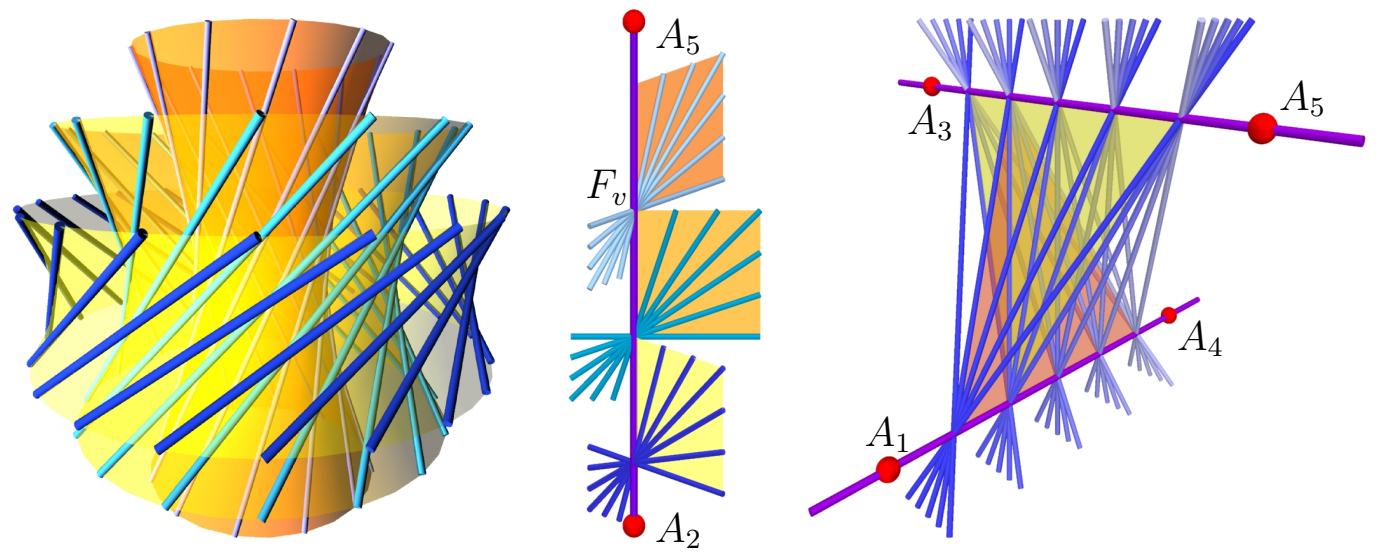

Figure 4: Three types of linear line congruences in $\mathbb{P}^{3}$. Left: the elliptic type. Middle: the parabolic type. Right: the hyperbolic type.

Case 3: The parameterization of $\Phi$ is assumed to be $\mathbf{f}(u, v)=\left(\frac{1}{2} u^{2}-\frac{1}{2} v^{2}, u v, u, v\right)$. Therefore rk $\left(\mathbf{a}_{1}, \mathbf{a}_{2}, \mathbf{a}_{4}\right)=3$ and $\mathbf{r k}\left(\mathbf{a}_{2}, \mathbf{a}_{3}, \mathbf{a}_{5}\right)=3$ and both $E_{u}$ and $E_{v}$ are linearly parameterized planes. The projective mapping $\alpha: E_{u} \rightarrow E_{v}$ maps the line of intersection $s=E_{u} \cap E_{v}$ onto itself. Since $\alpha$ restricted to $s=\alpha(s)$ has two conjugate complex fixed points, $\mathcal{G}$ is an elliptic linear line congruence joining points $F_{u}=\left(u \mathbf{a}_{1}+v \mathbf{a}_{2}+\mathbf{a}_{4}\right)$ and $F_{v}=\left(u \mathbf{a}_{2}-v \mathbf{a}_{1}+\mathbf{a}_{5}\right)$. The Plücker coordinates of lines $g$ are $\mathbf{G}=\left(u^{2}+v^{2}, v, u, 1,-v,-u\right) \mathbb{R}$ and satisfy the two linear relations $G_{2}+G_{5}=0, G_{3}+G_{6}=0$.

The Plücker coordinates of the axes are $(0,1, i, 0,1, i) \mathbb{R}$ and $(0,1,-i, 0,1,-i)$. The family $\mathcal{H}=\lambda(\mathcal{G})$ of polar lines is again an elliptic linear line congruence.

Case 4: The parameterization of $\Phi$ is assumed to be $\mathbf{f}(u, v)=\left(\frac{1}{2} u^{2}, \frac{1}{2} v^{2}, u v, u\right)$. Therefore rk $\left(\mathbf{a}_{1}, \mathbf{a}_{2}, \mathbf{a}_{4}\right)=3$ and $\mathbf{r k}\left(\mathbf{a}_{2}, \mathbf{a}_{3}, \mathbf{a}_{5}\right)=2$. The lines $g$ join points $F_{u}=\left(u \mathbf{a}_{1}+v \mathbf{a}_{2}+\mathbf{a}_{4}\right)$ and $F_{v}=\left(u \mathbf{a}_{2}+v \mathbf{a}_{3}\right)$. The family $\mathcal{G}$ decomposes into pencils of lines with vertices $F_{v}$ lying in planes spanned by $F_{v}, A_{4}=\left(0, \mathbf{a}_{4}\right) \mathbb{R}$ and $(0, u, 0, v, 0) \mathbb{R}$. These planes envelope a quadratic cone with equation $Q: y_{1} y_{2}-y_{3}^{2}=0, y_{0}=0$ and vertex $A_{4}$. The plane $E_{u}$ is a tangent plane of $Q$, the line $E_{v}$ is a tangent line of $Q$.

The Plücker coordinates of the lines $g$ are $\mathbf{G}=\left(u v, u^{2}, 0,-u, v,-v^{2}\right) \mathbb{R}$ and satisfy the algebraic equations $G_{3}=0, G_{1} G_{5}-G_{4} G_{6}=0$. The family of lines consists of those tangent lines of $Q$ which intersect the tangent line $E_{v}$. Applying the polarity $\lambda$ with respect to $\Omega$, the quadratic cone is mapped to a conic $C$ and the tangent line 

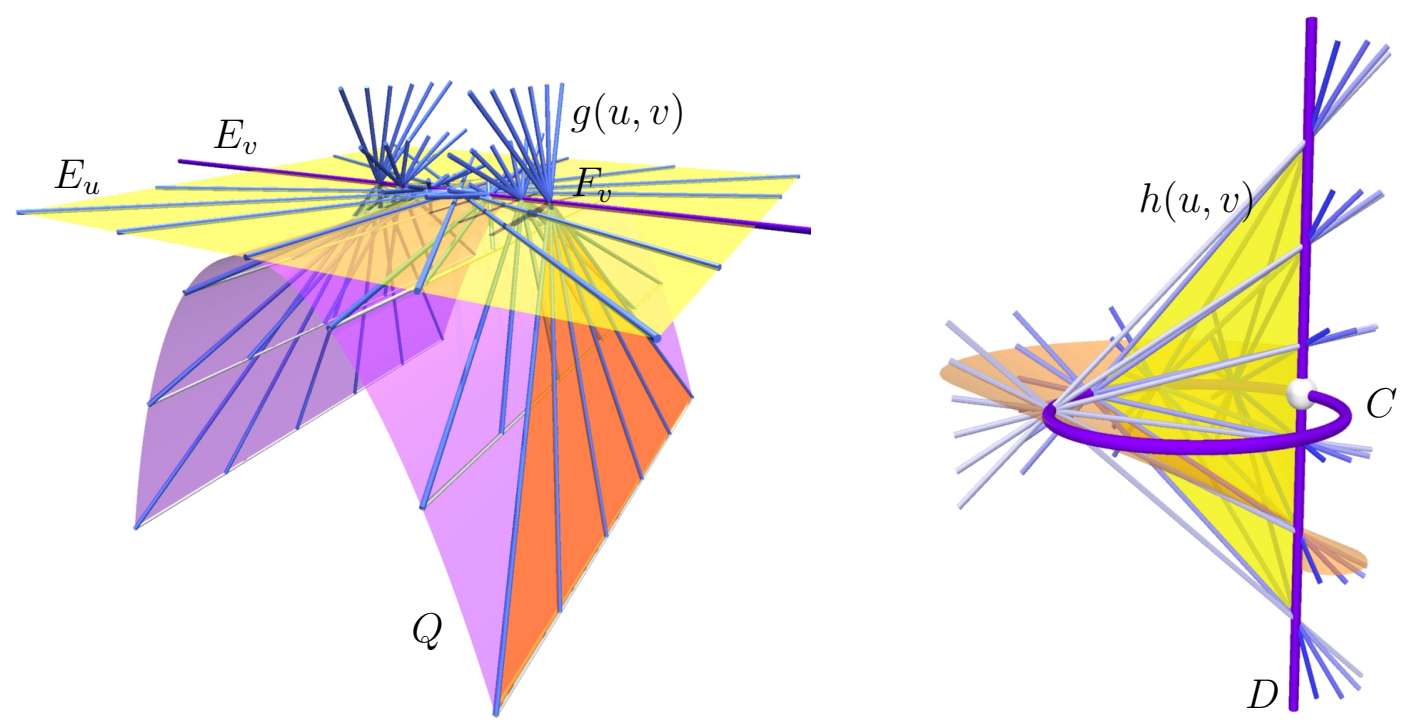

Figure 5: Left: Family of lines $\mathcal{G}$ from case 4. Right: Family of lines $\mathcal{H}$ from case 4.

is mapped to a line $D$, intersecting $C$ at one point. Thus the family of polar lines $\mathcal{H}=\lambda(\mathcal{G})$ consists of the lines $h(u, v)$ meeting $C$ and $D$, see Fig. 5.

Case 5: The parameterization of $\Phi$ is assumed to be $\mathbf{f}(u, v)=\left(\frac{1}{2} u^{2}+v, \frac{1}{2} v^{2}, u v, u\right)$. Therefore rk $\left(\mathbf{a}_{1}, \mathbf{a}_{2}, \mathbf{a}_{4}\right)=3$ and rk $\left(\mathbf{a}_{2}, \mathbf{a}_{3}, \mathbf{a}_{5}\right)=3$ and both $E_{u}$ and $E_{v}$ are linearly parameterized planes. The family $\mathcal{G}$ consists of lines joining the points $F_{u}=\left(u \mathbf{a}_{1}+v \mathbf{a}_{2}+\mathbf{a}_{4}\right)$ and $F_{v}=\left(u \mathbf{a}_{2}+v \mathbf{a}_{3}+\mathbf{a}_{5}\right)$ which are related by the projective mapping $\alpha: E_{u} \rightarrow E_{v}$. The Plücker coordinates of the lines $g$ are $\mathbf{G}=\left(u v, u^{2}-v,-1,-u, v,-v^{2}\right) \mathbb{R}$ and satisfy the relations $G_{1} G_{5}-G_{4} G_{6}=0$ and $G_{1} G_{3}-G_{4} G_{5}=0$.

The family $\mathcal{H}=\lambda(\mathcal{G})$ of polar lines $h$ is known to be the chordal variety of a spatial cubic $C$, see Fig. 6 . This can be verified by applying the polarity $\lambda: \mathbb{P}^{3} \rightarrow \mathbb{P}^{3 \star}$ which maps planes $E_{u}, E_{v}$ to points $S=\lambda\left(E_{u}\right), T=\lambda\left(E_{v}\right)$. Points and lines in $E_{u}$ and $E_{v}$ are mapped to planes and lines passing through $S$ and $T$. Let $\alpha^{\star}$ be the transformed projective mapping between points (stars) $S$ and $T$. As $\mathcal{G}$ consists of lines joining corresponding points $F_{u}$ and $F_{v}$, the family of polar lines $\mathcal{H}$ consists of the intersection lines $h=\varphi_{u} \cap \varphi_{v}$, where $\varphi_{u}=\lambda\left(F_{u}\right)$ and $\varphi_{v}=\lambda\left(F_{v}\right)$.

- The singular set of $\mathcal{H}$ is the spatial cubic $C$ and through any point of $C$ passes a quadratic cone $\Delta$ of chords of $\mathcal{H}$. Dual to that the singular set of $\mathcal{G}$ is a developable surface $D=\lambda(C)$ of class three.

- As the chords of the chordal variety $\mathcal{H}$ join two, not necessarily real points of a spatial cubic $C$, the lines $g \in \mathcal{G}$ are obtained as intersection lines of two tangent planes $\varepsilon(u)$ and $\varphi(v)$ of the developable surface $D=\lambda(C)$. The family $\mathcal{G}$ will be called axes variety.

- Besides the singular set, any point $X \notin C$ lies on exactly one chord of $\mathcal{H}$. Dual 

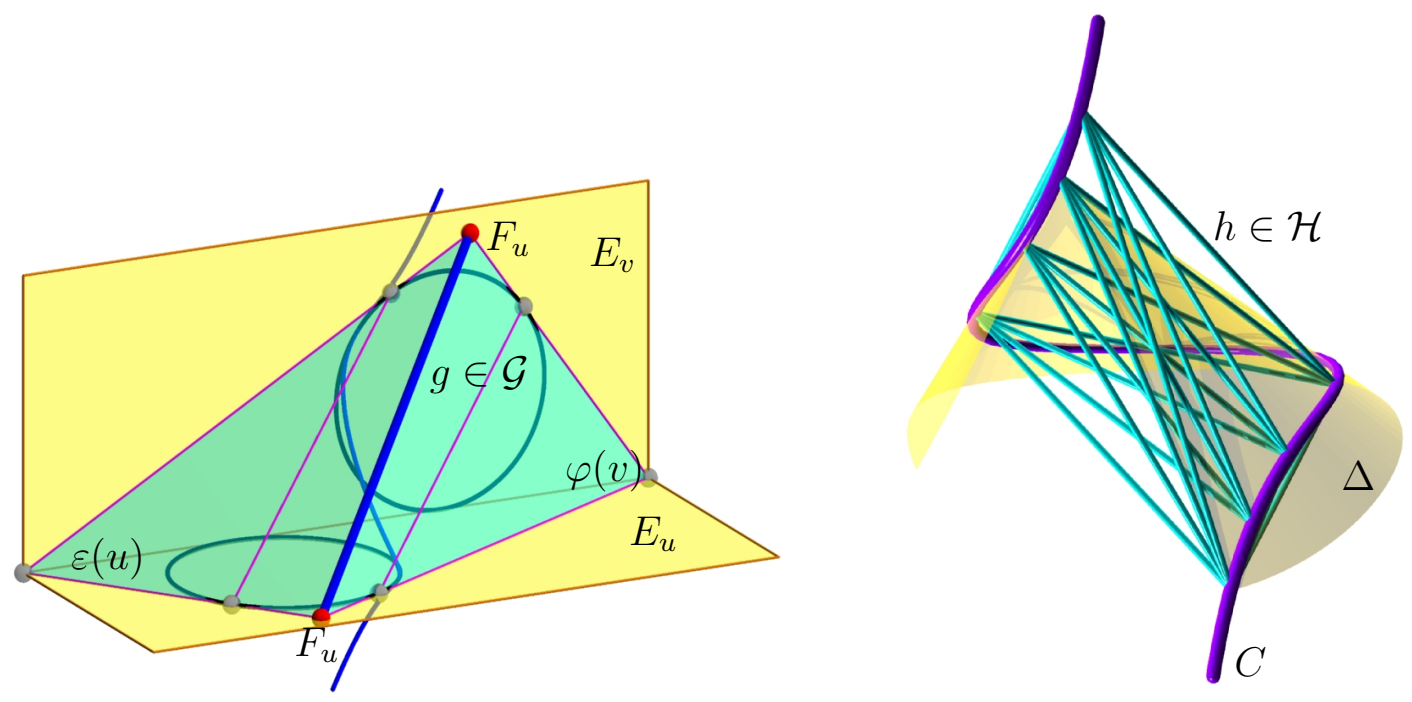

Figure 6: Left: Axis variety $\mathcal{G}$. Right: Chordal variety $\mathcal{H}$ of a spatial cubic $C$.

to that property, any plane $\varepsilon \in \omega$ not being tangent to developable surface $D$ carries exactly one line $g \in \mathcal{G}$.

Lemma 1 Let $\mathcal{H}$ be family of ideal lines of the normal planes $N$ of a quadratic triangular Bézier surface $\Phi$ in $\mathbb{R}^{4}$. Then $\mathcal{H}$ is either a linear line congruence (cases 1,2,3), or the lines meeting a conic $C$ and a line $D$ which intersects $C$ in a single point (case 4 ) or $\mathcal{H}$ is the chordal variety of a spatial cubic (case 5). In any of these cases $\mathcal{H}$ is a fibration of the 3-space $\omega$ at infinity.

Remark: Consider a quadratic triangular Bézier surface $\Phi$ which is contained in a 3dimensional subspace of $\mathbb{R}^{4}$. The family $\mathcal{G}$ of ideal lines $g$ of $\Phi$ 's tangent planes forms a ruled plane $E$. The polarity $\lambda$ maps $g \in E$ to lines $h$ passing through the point $\lambda(E)$ and obviously $\mathcal{H}$ is a star of lines. Even in this case the ideal lines $h$ of $\Phi$ 's normal planes are a fibration of $\omega$.

\section{The cyclographic image of quadratic triangular Bézier surfaces}

Let $\Phi$ be a quadratic triangular Bézier surface in $\mathbb{R}^{4}$, represented by the homogeneous coordinates $\mathbf{f}(u, v) \mathbb{R}=(1, \mathbf{s}(u, v))$, and let $g=F_{u} \vee F_{v}$ be the ideal lines of $\Phi$ 's tangent planes $T$. The polarity $\lambda: \mathbb{P}^{3} \rightarrow \mathbb{P}^{3 \star}$ with respect to $\Omega$ maps lines $g \in \omega$ to the ideal 
lines $h=\lambda(g) \in \omega$ of $\Phi$ 's normal planes $N$. According to Lemma 1 the family $\mathcal{H}$ forms a fibration of $\omega$. The construction of the cyclographic image $\gamma(\Phi)$ is performed as follows:

- We consider an arbitrary rational parameterization $\mathbf{w}(s, t)$ of the quadric $\Omega \in \omega$. Since $\Omega$ defines the scalar product (5) it follows $\langle\mathbf{w}, \mathbf{w}\rangle=0$. For practical reasons we may use the quadratic parameterization

$$
\mathbf{w}(s, t)=\left(2 s, 2 t, 1-s^{2}-t^{2}, 1+s^{2}+t^{2}\right) \in \mathbb{R}^{4} .
$$

- According to Lemma 1 there exists a unique line $h \in \mathcal{H}$ passing through any point $W=\mathbf{w} \mathbb{R} \in \Omega$ but $W \notin C$. Let $\mathbf{H}(u, v)=\left(H_{1}, \ldots, H_{6}\right)(u, v)$ be the Plücker coordinates of the lines $h \in \mathcal{H}$. The incidence relation of the lines $h$ and the points $W \in \Omega$ reads

$$
\left[\begin{array}{cccc}
0 & H_{4} & H_{5} & H_{6} \\
-H_{4} & 0 & H_{3} & -H_{2} \\
-H_{5} & -H_{3} & 0 & H_{1} \\
-H_{6} & H_{2} & -H_{1} & 0
\end{array}\right] \cdot \mathbf{w}(s, t)=\left[\begin{array}{l}
0 \\
0 \\
0 \\
0
\end{array}\right]
$$

Since the coordinates $H_{i}$ of lines $h$ satisfy the Plücker relation (3), the coefficient matrix has rank 2 and only two of four equations are relevant.

- According to Lemma 1 this polynomial system (14) has a unique solution

$$
u=a(s, t), \quad v=b(s, t),
$$

with rational functions $a$ and $b$, representing the reparameterization of the surface $\Phi$ in order to construct the cyclographic image linearly.

- Performing the reparameterization (15) results in $\mathbf{f}(s, t)$. Joining points $F=\mathbf{f}(s, t) \mathbb{R}$ with $W \in \Omega$, we obtain a system of isotropic projection lines

$$
i(s, t): \mathbf{f}(s, t)+\lambda \mathbf{w}(s, t), \text { with } \lambda \in \mathbb{R} .
$$

The intersection points $P(s, t)=i(s, t) \cap \mathbb{R}^{3}$ of $i(s, t)$ with $\mathbb{R}^{3}: x_{4}=0$ are contained in the cyclographic image $\gamma(\Phi)$ and $P(s, t)$ is a rational parameterization of $\gamma(\Phi)=$ $\gamma(\mathbf{f}(s, t))$.

- The offset of the envelope surface $\gamma(\Phi)$ at distance $d$ is a rational surface since it is obtained by intersecting the projection lines (16) with the 3 -space $x_{4}=d$.

This construction proves the following important result: 
Theorem 2 Let $\Phi$ be a quadratic triangular Bézier surface in $\mathbb{R}^{4}$ and let $S_{\Phi}$ be the corresponding two-parameter family of spheres in $\mathbb{R}^{3}$. The cyclographic image $\gamma(\Phi)$, which is the envelope of $S_{\Phi}$ is rational and the reparameterization (15) leads to a rational parameterization of $\gamma(\Phi)$. Moreover all offset surfaces of the envelope $\gamma(\Phi)$ are also rational.

Remark: The reparameterization (15) can also be obtained by replacing equation (14) by the equations

$$
\left\langle\mathbf{w}, \mathbf{f}_{u}\right\rangle=0,\left\langle\mathbf{w}, \mathbf{f}_{v}\right\rangle=0
$$

which are linear in $u$ and $v$ because $\mathbf{f}(u, v)$ is quadratic. Since $\langle\mathbf{w}, \mathbf{w}\rangle=0$ holds, we are looking for those isotropic vectors $\mathbf{w}$ which are orthogonal to $\mathbf{f}_{u}$ and $\mathbf{f}_{v}$, and thus span the normal plane $N$. This is also equivalent to solve (10).

We give two examples to demonstrate the computation of rational parameterizations of the cyclographic image $\gamma(\Phi)$ of a quadratic triangular Bézier surface $\Phi$.

1. Let $\Phi$ be parameterized by $\mathbf{f}(u, v)=\left(\frac{1}{2}\left(u^{2}-v^{2}\right), u v, u, v\right)$, corresponding to case 3 in section 2.3. The partial derivatives are $\mathbf{f}_{u}=(u, v, 1,0)$ and $\mathbf{f}_{v}=(-v, u, 0,1)$. The families $\mathcal{G}$ and $\mathcal{H}$ of ideal lines $g(u, v)$ and $h(u, v)$ are represented by

$$
\mathbf{G}(u, v)=\left(u^{2}+v^{2}, v, u, 1,-v,-u\right), \quad \mathbf{H}(u, v)=\left(-1, v,-u, u^{2}+v^{2}, v,-u\right) .
$$

We use (13) as parameterization of $\Omega$. Solving the system (14) leads to the reparameterization (15),

$$
u=\frac{\left(s^{2}+t^{2}\right)(t+s)-s+t}{2\left(s^{2}+t^{2}\right)}, \quad v=\frac{\left(s^{2}+t^{2}\right)(t-s)+s+t}{2\left(s^{2}+t^{2}\right)} .
$$

By intersecting the system of isotropic projection lines $i(s, t): \mathbf{f}(s, t)+\lambda \mathbf{w}(s, t)$ with $x_{4}=0$ we obtain a rational parameterization $\mathbf{p}(s, t)$ of the envelope $\gamma(\Phi)$. Offset surfaces of $\gamma(\Phi)$ at distance $d$ are obtained by intersecting the isotropic lines with $x_{4}=d$. The projection $\pi(\Phi)$ as well as the cyclographic image $\gamma(\Phi)$ are illustrated in Fig. 7.

2. Let $\Phi$ be parameterized by $\mathbf{f}(u, v)=\left(\frac{1}{2} u^{2}-v, \frac{1}{2} v^{2}, u v, u\right)$, corresponding to case 5 in section 2.3. The partial derivatives are $\mathbf{f}_{u}=(u, 0, v, 1)$ and $\mathbf{f}_{v}=(1, v, u, 0)$. The families $\mathcal{G}$ and $\mathcal{H}$ of ideal lines $g(u, v)$ and $h(u, v)$ are represented by

$$
\mathbf{G}(u, v)=\left(u v, u^{2}-v,-1,-u, v,-v^{2}\right), \quad \mathbf{H}(u, v)=\left(u,-v,-v^{2}, u v, u^{2}-v, 1\right) .
$$

We use (13) as parameterization of $\Omega$. Solving the system (14) leads to the reparameterization (15),

$$
u=2 \frac{\left(s^{2}+t^{2}\right)(s-t)-s-t}{\left(1-s^{2}-t^{2}\right)^{2}-4 s t}, \quad v=\frac{1+4 s^{2}-\left(s^{2}+t^{2}\right)^{2}}{\left(1-s^{2}-t^{2}\right)^{2}-4 s t} .
$$

By intersecting the system of isotropic projection lines $i(s, t): \mathbf{f}(s, t)+\lambda \mathbf{w}(s, t)$ with $x_{4}=0$ we obtain a rational parameterization $\mathbf{p}(s, t)$ of the envelope $\gamma(\Phi)$. The projection $\pi(\Phi)$ as well as the cyclographic image $\gamma(\Phi)$ are illustrated in Fig. 7. 

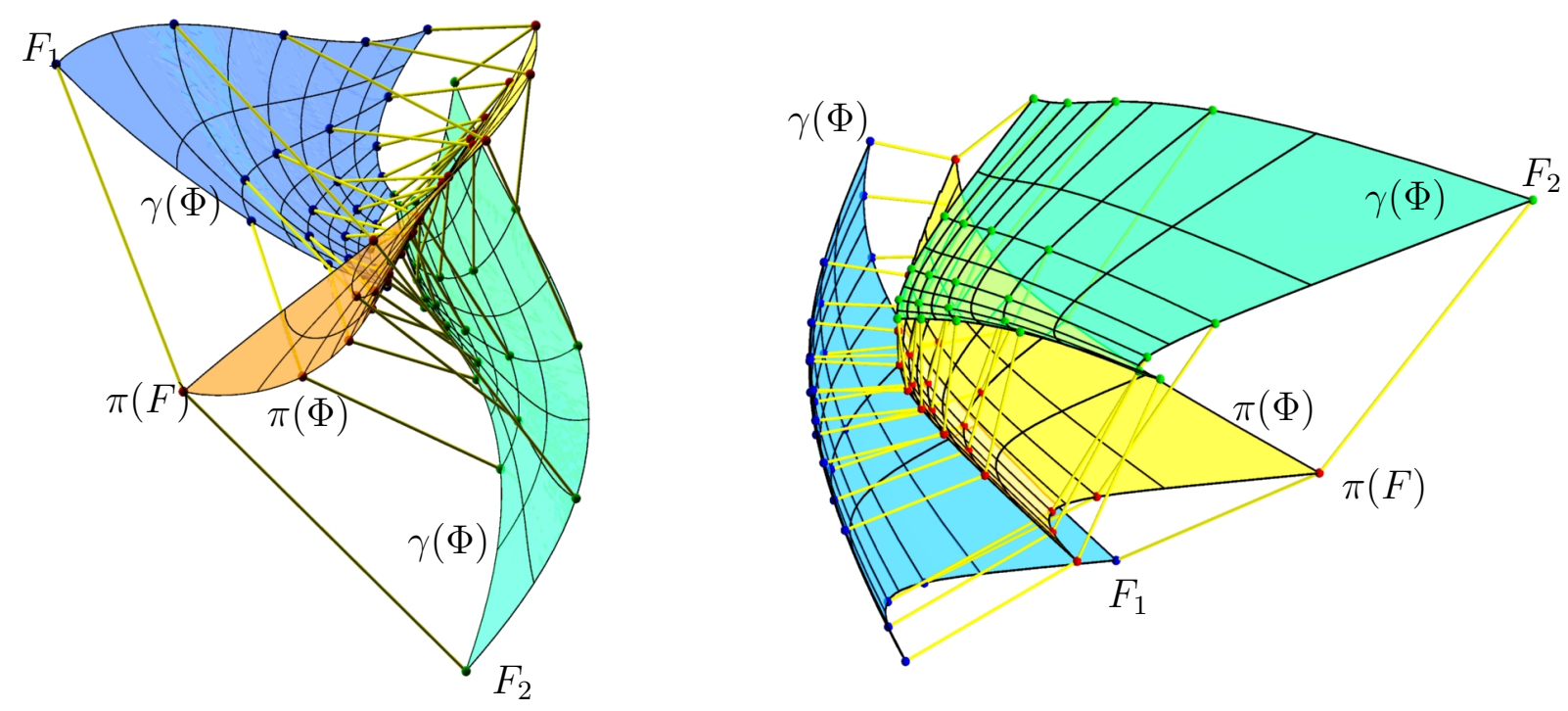

Figure 7: Cyclographic images $\gamma(\Phi)$ and orthogonal projection $\pi(\Phi)$ of quadratic triangular Bézier surfaces $\subset \mathbb{R}^{4}$. Left: Example 1. Right: Example 2.

\section{Generalizations of quadratic triangular Bézier sur- faces}

The rationality of the cyclographic image $\gamma(\Phi)$ of a quadratic triangular Bézier surface $\Phi$ with parameterization (11) depends only on the property that the family $\mathcal{H}$ of the ideal lines $h(u, v)$ of $\Phi$ 's normal planes $N$ is a fibration of the 3 -space $\omega$ at infinity.

A possible generalization interprets a surface $\Psi \subset \mathbb{R}^{4}$ as envelope of its two-parameter family of tangent planes $T(u, v)$. We require that the family $\mathcal{H}$ of ideal lines $h=N \cap \omega$ of $\Psi$ 's normal planes $N(u, v)$ is a fibration of $\omega$.

The ideal lines $g(u, v)=T \cap \omega$ of $\Psi$ 's tangent planes form the family $\mathcal{G}$ and are represented as lines of intersection of two planes

$$
\begin{array}{ll}
\varepsilon(u, v): & e_{1}(u, v) y_{1}+e_{2}(u, v) y_{2}+e_{3}(u, v) y_{3}+e_{4}(u, v) y_{4}=0 \\
\varphi(u, v): & f_{1}(u, v) y_{1}+f_{2}(u, v) y_{2}+f_{3}(u, v) y_{3}+f_{4}(u, v) y_{4}=0
\end{array}
$$

where $y_{i}$ denote homogeneous coordinates in $\omega$. The tangent planes $T$ of $\Psi$ are represented as intersection of 3-spaces $E(u, v)$ and $F(u, v)$ satisfying $E \cap \omega=\varepsilon$ and $F \cap \omega=\varphi$. Prescribing two rational functions $a(u, v)$ and $b(u, v), E$ and $F$ are determined by

$$
\begin{aligned}
& E(u, v): e_{1}(u, v) x_{1}+e_{2}(u, v) x_{2}+e_{3}(u, v) x_{3}+e_{4}(u, v) x_{4}=\mathbf{e}(u, v)^{T} \cdot \mathbf{x}=a(u, v), \\
& F(u, v): f_{1}(u, v) x_{1}+f_{2}(u, v) x_{2}+f_{3}(u, v) x_{3}+f_{4}(u, v) x_{4}=\mathbf{f}(u, v)^{T} \cdot \mathbf{x}=b(u, v),
\end{aligned}
$$

where $\mathbf{e}=\left(e_{1}, \ldots, e_{4}\right), \mathbf{f}=\left(f_{1}, \ldots, f_{4}\right)$ and $\mathbf{x}=\left(x_{1}, \ldots, x_{4}\right)$. We assume that the system 
of linear equations

$$
\begin{aligned}
& E: \mathbf{e}^{T} \cdot \mathbf{x}=a, \quad F: \mathbf{f}^{T} \cdot \mathbf{x}=b, \\
& E_{u}: \mathbf{e}_{u}^{T} \cdot \mathbf{x}=a_{u}, \quad F_{u}: \mathbf{f}_{u}^{T} \cdot \mathbf{x}=b_{u}, \\
& E_{v}: \mathbf{e}_{v}^{T} \cdot \mathbf{x}=a_{v}, \quad F_{v}: \mathbf{f}_{v}^{T} \cdot \mathbf{x}=b_{v},
\end{aligned}
$$

has a (unique) solution $\mathbf{p}(u, v)$. To guarantee that the family of ideal lines $T \cap \omega$ of $\Psi$ 's tangent planes $T$ coincides with $\mathcal{G}$, we prove that the points at infinity $P_{u}=\left(0, \mathbf{p}_{u}\right) \mathbb{R}$ and $P_{v}=\left(0, \mathbf{p}_{v}\right) \mathbb{R}$ satisfy $P_{u} \vee P_{v}=\varepsilon \cap \varphi=g(u, v)$.

Differentiating $\mathbf{e}^{T} \cdot \mathbf{p}=a$ with respect to $u$ and $v$ and taking $\mathbf{e}_{u}^{T} \cdot \mathbf{p}=a_{u}$ and $\mathbf{e}_{v}^{T} \cdot \mathbf{p}=a_{v}$ into account, leads to

$$
\mathbf{e}^{T} \cdot \mathbf{p}_{u}=0, \text { and } \mathbf{e}^{T} \cdot \mathbf{p}_{v}=0 .
$$

This expresses $g=P_{u} \vee P_{v} \in \varepsilon$. Analogously by differentiating $\mathbf{f}^{T} \cdot \mathbf{p}=b$ it follows that $g=P_{u} \vee P_{v} \in \varphi$. This proves that the tangent planes $T(u, v)$ of the surface $\Psi$ pass through the intersection $g=\varepsilon \cap \varphi=T \cap \omega$.

The question arises, which conditions have to be fulfilled by the functions $\mathbf{e}(u, v), \mathbf{f}(u, v)$ and $a(u, v), b(u, v)$ such that the family of planes $T(u, v)=E(u, v) \cap F(u, v)$ envelope a surface $\Psi$ ? It can be proved that if $\mathcal{H}$ is a fibration of $\omega$, then the coefficient matrix of (20) has rank 4. We list the possible cases and describe how to choose planes $\varepsilon(u, v)$ and $\varphi(u, v)$ to generate $g=\varepsilon \cap \varphi$.

- If $\mathcal{H}$ is a star of lines, $\mathcal{G}$ is a ruled plane and $g(u, v)$ is obtained by intersecting the planes of a star $\varepsilon(u, v)$ with the fixed carrier plane $\varphi$ of $\mathcal{G}$.

- If $\mathcal{H}$ is a hyperbolic linear line congruence with axes $A$ and $B$, the lines $g(u, v)$ are the intersection lines of two pencils of planes $\varepsilon(u)$ and $\varphi(v)$ passing through $\lambda(A)$ and $\lambda(B)$, respectively.

- If $\mathcal{H}$ is a parabolic linear line congruence with axes $A$, the lines $g(u, v)$ are the intersection lines of the pencil of planes $\varepsilon(u)$ through $\lambda(A)$ and an appropriate pencil of planes $\varphi(u, v)$ passing through a point $\mathbf{v}(u) \in \lambda(A)$. The correspondence $\varepsilon(u) \mapsto$ $\mathbf{v}(u)$ is a projective mapping.

- If $\mathcal{H}$ is an elliptic linear line congruence, the lines $g(u, v)$ are the intersection lines of two stars of planes $\varepsilon(u, v)$ and $\varphi(u, v)$ with vertices $E$ and $F$, respectively. The mapping $\varepsilon \mapsto \varphi$ keeps the pencil of planes through $E \vee F$ fixed as a whole.

- If $\mathcal{H}$ is the chordal variety of a spatial cubic $C, \mathcal{G}$ is the axes variety of a developable surface $D$ of class 3 whose tangent planes are $\varepsilon(t)$. The axis variety $\mathcal{G}$ consists of lines $g(u, v)=\varepsilon(u) \cap \varphi(v)$ with $\varphi(v)=\varepsilon(v)$, just by substituting $t=u$ and $t=v$ in $\varepsilon(t)$. 
- If $\mathcal{H}$ consists of all lines meeting a degree $n$-curve $C$ and a line $L$ which meets $C$ in $n-1$ points, $\mathcal{G}$ consists of those tangent lines of $D=\lambda(C)$ which meet the line $\lambda(L)$. We can represent $g(u, v)$ as intersection lines of the tangent planes $\varepsilon(u)$ of $D$ and the pencil of planes $\varphi(v)$ passing through the line $\lambda(L)$.

For each of the discussed cases functions $a(u, v)$ and $b(u, v)$ can be chosen such that this system has a unique solution. For particular cases, like the hyperbolic linear line congruence or the chordal variety, $\mathbf{e}$ and $\mathbf{f}$ are univariate rational vector functions and $a$ and $b$ can be chosen as arbitrary univariate rational functions. Only in the cases where $\mathcal{H}$ is a parabolic or an elliptic linear line congruence $a$ and $b$ have to satisfy certain conditions. We summarize the obtained results.

Theorem 3 Let a two-parameter family $\mathcal{H}$ of lines $h$ be a fibration of the 3-space at infinity $\omega \subset \mathbb{P}^{4}$ and let $\mathcal{G}$ be the family of polar lines $g$ with respect to $\Omega$. The families $\mathcal{G}$ and $\mathcal{H}$ are interpreted as ideal lines of tangent planes $T$ and normal planes $N$ of a surface $\Psi$. Considering $\Psi$ as envelope of its tangent planes $T$, one has to choose appropriate rational functions $a(u, v)$ and $b(u, v)$ in order to determine 3-spaces $E$ and $F$ with $T=E \cap F$. A rational parameterization $\mathbf{p}(u, v)$ of $\Psi$ is obtained as solution of the system (20), or any algebraically equivalent one.

Remark: The parameterization $\mathbf{p}(u, v)$ as solution of the system (20) is a general rational representation of $\Psi$. In order to construct rational parameterizations of the cyclographic image $\gamma(\Psi)$ one has to perform a reparameterization based on the system (14) analogously as in the case of quadratic triangular Bézier surfaces in Section 3.

An example shall illustrate the method. The ideal lines $g$ of the tangent planes of a surface $\Psi$ to be determined are the lines of intersection of tangent planes $\varepsilon(u): x_{1}+u x_{2}+u^{2} x_{3}+$ $u^{3} x_{4}=0$ of a developable surface $D$ of class 3 and a pencil of planes $\varphi(v): x_{1}+v x_{4}=0$ passing through a line of $L^{\prime}$ axes variety. The polar lines $h$ form the family $\mathcal{H}$ of lines meeting the cubic $C=\lambda(D)$ and the chord $\lambda(L)$ of $C$. The parameterization of $C$ is $\mathbf{c}(u)=\left(1, u, u^{2},-u^{3}\right)$. The univariate support functions are chosen as $a(u)=1+u^{2}$ and $b(v)=1+v^{2}$.

The families $\mathcal{G}$ and $\mathcal{H}$ of ideal lines $g(u, v)$ and $h(u, v)$ are represented by

$$
\mathbf{G}(u, v)=\left(u^{2} v,-u v, 0,-u,-u^{2}, v-u^{3}\right), \quad \mathbf{H}(u, v)=\left(-u,-u^{2},-v+u^{3},-u^{2} v, u v, 0\right) .
$$

Solving (20) gives the parameterization $\mathbf{f}(u, v)$ of $\Psi$,

$$
\mathbf{f}(u, v)=\left(v^{2}-1, \frac{-2\left(u^{3}+v\right)}{u v}, \frac{v^{2}+4 u^{3} v-u^{2}}{u^{2}},-2 v\right) .
$$

Using (13) as parameterization of $\Omega$, the solution of the system (14) leads to the rational reparameterization

$$
u=\frac{-1}{2 t\left(-1+s^{2}+t^{2}\right)}, v=\frac{\left(t^{4}+2 t^{3}+2\left(s^{2}-1\right) t^{2}+2\left(1+s^{2}\right) t+\left(s^{2}-1\right)^{2}\right)\left(1-s^{2}-t^{2}\right)}{4 t\left(-s+s^{3}+t^{2} s+2 t^{2}\right)} .
$$


The reparameterized surface $\mathbf{f}(s, t)$ is used to form the the system of isotropic projection lines $i(s, t): \mathbf{f}(s, t)+\lambda \mathbf{w}(s, t)$. The intersection points $\mathbf{p}(s, t)$ of $i(s, t)$ with $x_{4}=0$ is a rational parameterization $\mathbf{p}(s, t)$ of the cyclographic image $\gamma(\Psi)$. Rational parameterizations of the offsets of $\gamma(\Psi)$ at distance $d$ are obtained by intersecting $i(s, t)$ with $x_{4}=d$. The projection $\pi(\Psi)$ as well as the cyclographic image $\gamma(\Psi)$ are illustrated in Fig. 8.

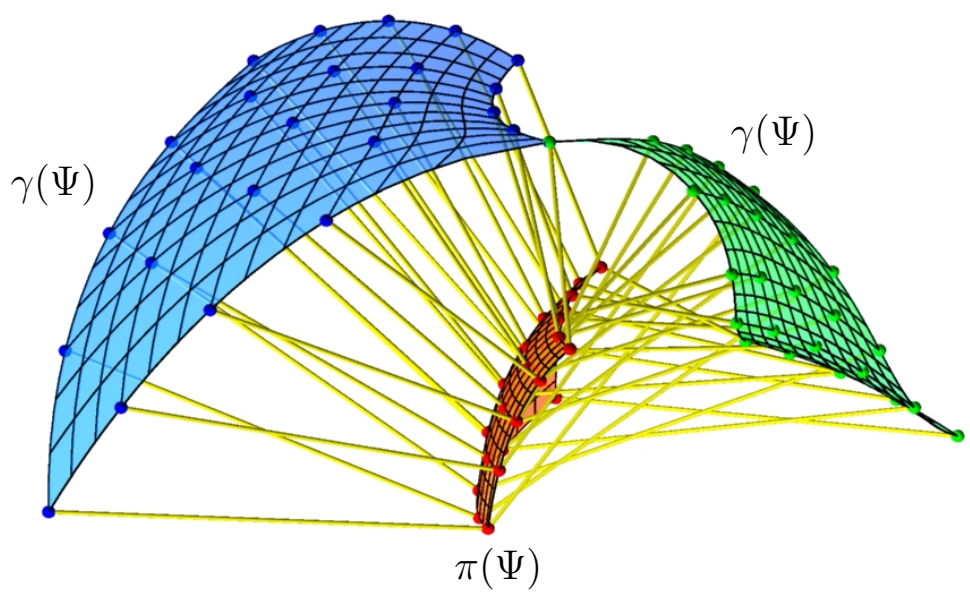

Figure 8: Rational envelope $\gamma(\Psi)$ and top view $\pi(\Psi)$ of a surface $\Psi \in \mathbb{R}^{4}$.

\section{Conclusion}

We have proved that the envelopes $\gamma(\Phi)$ of two-parameter families of spheres in $\mathbb{R}^{3}$ corresponding to quadratic triangular Bézier surfaces $\Phi$ in $\mathbb{R}^{4}$ are rational. Explicit parameterizations of $\gamma(\Phi)$ are given. The rationality of the offsets of $\gamma(\Phi)$ is a direct consequence of the construction. Further we have pointed to a possible generalization of this result and have outlined the strategy to construct surfaces with similar properties. A detailed discussion is postponed to another contribution.

\section{Acknowledgments}

This work has been funded in parts by the Austrian Science Fund FWF within the research network S92. Moreover, part of this research has been carried out through a research stay of the first author at the University of Siena. The authors thank Bert Jüttler for his hint concerning the reparameterization (17). 


\section{References}

[1] Albrecht, G., 2002. The Veronese surface revisited, J. Geom. 73, 22-38.

[2] Apery, F., 1987. Models of the Real Projective Plane: Computer Graphics of Steiner and Boy Surfaces. Vieweg, Braunschweig.

[3] Coffmann, A., Schwartz, A.J., Stanton, C, 1995. The algebra and geometry of Steiner and other quadratically parameterizable surfaces, Comp. Aided Geom. Design 13 (1996), $257-286$.

[4] Degen, W.L.F., 1994. The Types of Triangular Bézier Surfaces, in: IMA Conference on the Mathematics of Surfaces, 153-170.

[5] Farouki,R. T. and Neff,C. A., 1990. Algebraic properties of plane offset curves Comp. Aided Geom. Design 7, 101-127.

[6] Farouki, R.T., 1992. Pythagorean-hodograph curves in practical use, in: Barnhill, R.E., ed., Geometry Processing for Design and Manufacturing, SIAM, Philadelphia, 3-33.

[7] Farouki, R.T. and Sakkalis, T., 1994. Pythagorean-hodograph space curves, Advances in Comp. Math. 2, 41-66.

[8] Farouki, R.T. and Neff, C.A., 1995. Hermite interpolation by Pythagorean hodograph quintics, Math. Comp. 64, 1589-1609.

[9] Farouki, R.T., 1996. The elastic bending energy of pythagorean-hodograph curves Comp. Aided Geom. Design 13, 227-241.

[10] Farouki, R.T., 2002. Pythagorean Hodograph curves, in: Handbook of Computer Aided Geometric Design, Farin, G., Hoschek, J., Kim, M.-S. eds.), Elsevier.

[11] Farouki, R.T., Manni, C. and Sestini, A., 2003. Spatial $C^{2}-\mathrm{PH}$ quintic splines, in: Curve and Surface Design: St. Malo 2002, (Lyche, T., Mazure, M.L. and Schumaker, L.L., eds.), Nashboro Press, 147-156.

[12] Farouki, R.T. and Sakkalis,T., 2007. Rational space curves are not 'unit speed', Comp. Aided Geom. Design 24, 238-240.

[13] Hoschek, J., Lasser, D., 1993. Fundamentals of Computer Aided Geometric Design. A. K. Peters, Wellesley, MA.

[14] Jüttler, B., 1998. Triangular Bézier surface patches with a linear normal vector field, in: The Mathematics of Surfaces VIII, Information Geometers, 431-446.

[15] Jüttler, B. and Sampoli, M.L., 2000. Hermite interpolation by piecewise polynomial surfaces with rational offsets, Comp. Aided Geom. Design 17, 361-385. 
[16] Jüttler, B., 2001. Hermite interpolation by Pythagorean hodograph curves of degree seven, Mathematics of Computation 70, 1089-1111.

[17] Kosinka, J. and Jüttler,B., 2006. $G^{1}$-Hermite interpolation by Minkowski Pythagorean hodograph cubics, Comp. Aided Geom. Design 23, 401-418

[18] Kosinka, J. and Jüttler, B., 2007. MOS surfaces: Medial surface transforms with rational domain boundaries, in: The Mathematics of Surfaces XII, R. Martin, M. Sabin, J. Winkler (eds.), Lecture Notes in Computer Science, Springer, to appear.

[19] Krasauskas, R., 2006. Minimal rational parameterization of canal surfaces, Computing, to appear.

[20] Krasauskas, R. and Mäurer, C., 2000. Studying cyclides with Laguerre geometry. Comp. Aided Geom. Design 17, 101-126.

[21] Lü, W. and Pottmann, H., 1996. Pipe surfaces with rational spine curve are rational, Comp. Aided Geom. Design 13, 621-628.

[22] Lü, W., 1994. Rationality of the offsets to algebraic curves and surfaces, Applied Mathematics 9 (Ser. B), 265-278.

[23] Lü, W., 1995. Offset-rational parametric plane curves, Comp. Aided Geom. Design 12, 601-616.

[24] Lü, W., 1996. Rational parameterization of quadrics and their offsets, Computing 57, $135-147$.

[25] Pelosi, F., Sampoli, M.L., Farouki, R.T., and Manni, C., 2007. A control polygon scheme for design of planar $C^{2}-\mathrm{PH}$ quintic spline curves Comp. Aided Geom. Design, 24, 28-52.

[26] Peternell, M. and Pottmann, H., 1998. A Laguerre geometric approach to rational offsets, Comp. Aided Geom. Design 15, 223-249.

[27] Peternell, M. and Odehnal, B.: Convolution surfaces of quadratic triangular Bézier surfaces, Comp. Aided Geom. Design, to appear.

[28] Peters, J., Reif, U., 1998. The 42 equivalence classes of quadratic surfaces in affine n-space, Comp. Aided Geom. Design 15, 459-473.

[29] Pottmann, H., 1995. Rational curves and surfaces with rational offsets, Comp. Aided Geom. Design 12, 175-192.

[30] Pottmann, H. (1995), Curve design with rational Pythagorean-hodograph curves, Advances in Comp. Math. 3, 147-170. 
[31] Pottmann, H., 1995. Studying NURBS curves and surfaces with classical geometry, in: Mathematical Methods for Curves and Surfaces, eds: M. Dæhlen and T. Lyche and L. L. Schumaker, Vanderbilt University Press, 413-438.

[32] Pottmann, H., Lü, W. and Ravani, B., 1996. Rational ruled surfaces and their offsets, Graphical Models and Image Processing 58, 544-552.

[33] Pottmann, H. and Peternell, M., 1998. Applications of Laguerre Geometry in CAGD, Comp. Aided Geom. Design 15, 165-186.

[34] Pottmann, H. and Wallner, J., 2001. Computational Line Geometry. Springer-Verlag.

[35] Sampoli, M.L., Peternell, M. and Jüttler, B., 2006. Exact parameterization of convolution surfaces and rational surfaces with linear normals, Comp. Aided Geom. Design 23, 179-192.

[36] Sederberg, T.W., Anderson, D.C., 1985. Steiner surface patches, IEEE Comp. Graphics \& Applications 5, 23-36.

[37] Š́r, Z., Feichtinger, R. and Jüttler, B., 2006. Approximating Curves and their Offsets using Baircs and Pythagorean hodograph Quintics, Computer Aided Design 38, 608618.

[38] Š́r, Z. and Jüttler, B., 2007. $C^{2}$-Hermite interpolation by Pythagorean hodograph space curves, Mathematics of Computation 76, 1373-1391.

[39] Steiner, J., 1882. Gesammelte Werke II, Berlin, 723-724, 741-742.

[40] Timar, S.D., Farouki, R.T., Smith, T.S. and Boyadjieff, C.L., 2005. Algorithms for timeoptimal control of CNC machines along curved tool paths, Robotics and ComputerIntegrated Manufacturing, 21, 37-53.

[41] Tsai, Y.-F., Farouki, R.T. and Feldman, B., 2001. Performance analysis of CNC interpolators for time-dependent feedrates along PH curves, Comp. Aided Geom. Design 18, 245-265.

[42] Zindler, K., 1921. Algebraische Liniengeometrie, in: Encykl. d. Math. Wiss. II, 2.2, 973-1228. 